\title{
CONSTRUCTION OF AN INNER FUNCTION IN THE LITTLE BLOCH SPACE
}

\author{
KENNETH STEPHENSON
}

\begin{abstract}
An explicit construction using Riemann surfaces and Brownian motion is given for an inner function in the unit disc which is not a finite Blaschke product yet belongs to the little Bloch space $\mathscr{B}_{0}$. In addition to showing how an inner function can meet the geometric conditions for $\mathscr{B}_{0}$, this example settles an open question concerning the finite ranges of inner functions: the values which it takes only finitely often are dense in the disc.
\end{abstract}

A function $f$ analytic on the unit disc $D=\{|z|<1\}$ belongs to the space $\mathscr{B}_{0}$, the so-called little Bloch space, if it satisfies the equality $\lim _{|z| \rightarrow 1}(1-|z|)\left|f^{\prime}(z)\right|=0$. There are several equivalent definitions, but for our purposes the most useful relates to the geometry of the image surface $\mathscr{R}_{f}$; namely, $f \in \mathscr{B}_{0}$ if and only if for each $\varepsilon>0$ there exists a compact set $K \subseteq \mathscr{R}_{f}$ so that $\mathscr{R}_{f}$ contains no schlicht discs of radius $\varepsilon$ centered at points outside of $K$. An inner function is an analytic function mapping the disc $D$ into itself whose radial limits are of modulus one almost everywhere on the unit circle $T$. It is evident that finite Blaschke products are inner functions belonging to $\mathscr{B}_{0}$; however, it would at first appear quite difficult for more general inner functions to belong. Indeed, their image surfaces cover nearly all values of the unit disc infinitely often outside of any compact subset, and at the same time their boundaries lie largely over the unit circle. Nonetheless, J. M. Anderson [2] saw that such an example was possible using certain positive singular measures on the unit circle first constructed by J.-P. Kahane. (See [5, 8, and 13, VII.5].) In [7], Donald Sarason asks for a direct construction of an infinite Blaschke product in $\mathscr{B}_{0}$, with the aim of characterizing the zero sets of such functions. He also writes of a suggestion of Tom Wolff concerning the Hausdorff measure of their singular sets.

The construction here is not of the type Sarason requests-it does not provide the desired zero set. Rather, we construct our function by building its image surface and checking the geometric condition above. Still, it does show how inner functions can overcome the geometric constraints for membership in $\mathscr{B}_{0}$, it satisfies the Hausdorff measure property suggested by Wolff, and it raises some other questions which may be of interest. Moreover, the particular example we obtain answers an open question regarding the finite ranges of inner functions.

A point $w \in D$ is in the finite range of an inner function $f$ if the cardinality of $f^{-1}\{w\}$ is finite. Of course, for a finite Blaschke product, the finite range is

Received by the editors November 21,1987 .

1980 Mathematics Subject ('lassification (1985 Revision). Primary 30D50; Secondary 30D40, $30 \mathrm{~F} 99$.

The author gratefully acknowledges the support of the National Science Foundation and the Science Alliance of Tennessee. 
the entire disc $D$; however, by a theorem of Frostman, for other inner functions the finite range is of (logarithmic) capacity zero. In studying the finite ranges of inner functions in [11], the author left open the question of whether the closure of the finite range was also of capacity zero. The answer is no, for the example in this paper has a finite range whose closure is the entire unit disc. In particular, this illustrates that an infinite Blaschke product need not take every value in some neighborhood of the origin infinitely often.

The author would like to thank Christian Pommerenke, Wayne Smith, and Tom Wolff for helpful conversations on the material presented here. The geometric characterization of $\mathscr{B}_{0}$ is given in Theorem A(ii) of [10]; for the basic notions regarding Brownian motion and Riemann surfaces which we use, see Davis [4] and Ahlfors and Sario [1], respectively.

1. An overview. Our method of construction depends on the Riemann mapping or uniformization theorem. We first construct a simply connected Riemann surface $\mathscr{R}$ by pasting together infinitely many copies of the unit disc, slit along specified radial segments. The natural projection $\pi$ of $\mathscr{R}$ to the unit disc is a bounded analytic function, so $\mathscr{R}$ is conformally equivalent to the unit disc by the uniformization theorem. Let $\phi: D \rightarrow \mathscr{R}$ be a conformal mapping. The function $f$ of interest is defined by

$$
f \equiv \pi \circ \phi: D \rightarrow D
$$

automatically we have $\mathscr{R}_{f}=\mathscr{R}$. The construction of $\mathscr{R}$ will guarantee that the range of $f$ has a dense set of points which are taken only finitely often. For a bounded analytic function in $D$, this alone implies the geometric conditions for membership in $\mathscr{B}_{0}$.

The proof that $f$ is an inner function is more delicate. It suffices to show that the full harmonic measure of $\mathscr{R}$ lies on a part of its ideal boundary which projects to the unit circle under $\pi$. Here is where Brownian motion arguments are useful. A subset of the ideal boundary of a surface has full harmonic measure if and only if almost every Brownian path, starting from some fixed point of $\mathscr{R}$, eventually exits the surface at a point of that subset. In our case, we show that almost every Brownian path has a projection under $\pi$ which converges to the unit circle.

2. The construction of $\mathscr{R}$. Let $E_{0} \subseteq E_{1} \subseteq E_{2} \subseteq \cdots$ be finite subsets of nonzero points in the unit disc, $E_{0} \neq \varnothing$. We require that their union be dense and that no two points of their union have the same argument. For each index $j$ we define the surface $\Omega_{j}$ obtained from a copy of the unit disc with the points of $E_{j}$ removed and with radial slits cut from those points to the unit circle. (See Figure 1.) The surface $\mathscr{R}$ will be obtained by pasting together many such "sheets" along opposite edges of common slits. All of the pasting operations are essentially the same, so let us describe one rather fully here at the beginning: Fix a point $e \in E_{n} \backslash E_{n-1}$ and denote by $\gamma$ the radial segment from $e$ to the unit circle. A sheet $\Omega_{k}$ has a slit along $\gamma$ if and only if $k \geq n$. Suppose $k, l \geq n$ and consider pasting $\Omega_{k}$ to $\Omega_{l}$ along $\gamma$. Of course, $\gamma$ has two sides which we might refer to as left and right. Say that we want to attach the left side of $\gamma$ on $\Omega_{k}$ to the right side of $\gamma$ on $\Omega_{l}$. We take the disjoint union of $\Omega_{k}$ and $\Omega_{l}$, identify these edges, and use the induced topology on this new surface. The natural projections, call them $\pi_{k}$ and $\pi_{l}$, of $\Omega_{k}$ 
and $\Omega_{l}$ to the unit disc agree on the overlap and provide a continuous, locally oneto-one projection to the disc. This induces a conformal structure on the new surface which agrees with the original structures on the subsurfaces corresponding to $\Omega_{k}$ and $\Omega_{l}$. The new surface is simply connected by van Kampen's theorem, since the original two pieces were simply connected and were joined along a simply connected set, namely $\gamma$. (Note that the new surface has points lying over the interior of the segment $\gamma$, but no point over e.) In general, we will be joining one new sheet to a surface already made up of several others, but the operation is the same: each connection is made from some edge of a slit $\gamma$ on one sheet to the opposite edge of $\gamma$ on another.

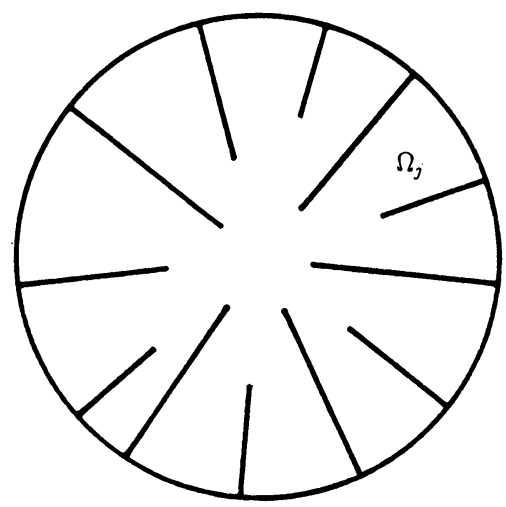

FIGURE 1

The surface $\mathscr{R}$ is the directed limit of a sequence of nested surfaces $\left\{\mathscr{R}_{j}\right\}$. The first of these, $\mathscr{R}_{0}$, is just $\Omega_{0}$, and has $2 c$ unattached edges formed by the slits to the points of $E_{0}$, where $c$ is the cardinality of $E_{0}$. The next surface, $\mathscr{R}_{1}$, will result from several additional stages of pastings: First, we paste $2 c$ copies of $\Omega_{1}$ to the $2 c$ edges of $\mathscr{R}_{0}$, obtaining a surface which has unattached edges formed by the slits in these copies of $\Omega_{1}$. The sheets we added at this stage will be called sheets of the first generation. Next, we paste copies of $\Omega_{1}$ to all of the new unattached edges-these are sheets of the second generation. We continue in this way until we have adding some integer number $g_{1}$ of generations to $\mathscr{R}_{0}$, all made of copies of $\Omega_{1}$, the appropriate number $g_{1}$ to be determined later. The surface we have built to this stage is now denoted $\mathscr{R}_{1}$. Observe that all the unattached edges of $\mathscr{R}_{1}$ are the ones left from the last generation of sheets we attached, since edges of all earlier generations have had additional sheets pasted on. The surface $\mathscr{R}_{2}$ is obtained likewise, attaching some number $g_{2}$ of generations of copies of $\Omega_{2}$ to the edges remaining on $\mathscr{R}_{1}$. One continues inductively in this way to obtain the sequence $\left\{\mathscr{R}_{j}\right\}$.

The $\mathscr{R}_{j}$ 's are nested simply connected surfaces lying over the unit disc. Their directed limit $\mathscr{R}$ is therefore a simply connected Riemann surface with a natural projection $\pi: \mathscr{R} \rightarrow D$. Note that there are no unattached edges in the limiting surface; all will have been pasted to succeeding generations during the construction. There are no points of $\mathscr{R}$ lying over points of $E_{0}$, for none of the sheets $\Omega_{j}$ contains points over $E_{0}$. The points of $E_{1} \backslash E_{0}$ are covered only once, by the original copy of 
$\Omega_{0}$. And in general, the points of $E_{n} \backslash E_{n-1}$ are covered only finitely often-namely, by the copies of $\Omega_{0}, \Omega_{1}, \ldots, \Omega_{n-1}$ which went into the construction of $\mathscr{R}_{n-1}$. We have met one of our objectives: There is a dense set in $D$ which $\mathscr{R}$ covers only finitely often, and consequently the function $f$ we obtain from $\mathscr{R}$ is in $\mathscr{B}_{0}$.

It may be helpful to identify this surface $\mathscr{R}$ with a tree whose nodes represent the copies of the $\Omega_{j}$ 's and whose edges represent the pastings. (See Figure 2.) Each node has two edges for every slit in its corresponding sheet. The base node corresponds to the single copy of $\Omega_{0}$ we started with and succeeding generations of nodes correspond to the generations of sheets we added. There are uncountably many trees (i.e., surfaces) we could build with similar methods, just by varying our selection of nodes at each generation; and we will actually use one other in our later work. We do not claim to understand fully how the properites of the trees and their corresponding surfaces are related, but this representation is suggestive. Observe, for instance, that in our tree if one stands at a node corresponding, say, to $\Omega_{n}$, then one must travel at least $g_{n+1}$ edges to reach a node corresponding to $\Omega_{n+2}$. We will be choosing the $g_{j}$ 's so that Brownian travelers on the surface will be discouraged from making such long trips.

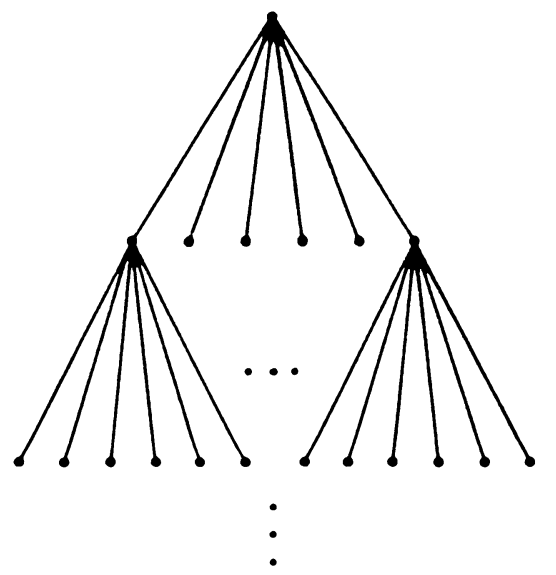

FIGURE 2

3. Proof that $f$ is an inner function. Let $w_{0} \in \mathscr{R}$ be the point lying over $z=0$ on the sheet $\Omega_{0}$ with which we started the construction of $\mathscr{R}$. For convenience, choose the Riemann mapping function $\phi: D \rightarrow \mathscr{R}$ so that $\phi(0)=w_{0}$; then $f(0)=\pi(\phi(0))=0$. Denote by $Z_{t}, t>0$, the standard Brownian motion in $D$ starting at $z=0$ and stopped on first hitting the unit circle. It is well known that $f$ has an asymptotic value along almost every Brownian path. It will generally be helpful to picture the Brownian motion, transplanted via $\phi$, as a motion on $\mathscr{R}$ starting at $w_{0}$. Thus, on $\mathscr{R}$ almost every Brownian path starting at $w_{0}$ will approach the ideal boundary and will give rise to an asymptotic value for $\pi$. To prove that $f$ is an inner function, it suffices to prove that these asymptotic values have modulus one on almost every Brownian path. Since it is not difficult to see that every point interior to the unit disc is an asymptotic value of $\pi$ along some path, our main task is to show that the positive integers $g_{j}$ used in the construction of $\mathscr{R}$ can be chosen so that asymptotic values interior to the disc are almost never taken on Brownian paths. 
We will choose the $g_{j}$ inductively, with $g_{0}=1$, corresponding with the fact that we used only one sheet $\Omega_{0}$ in our construction of $\mathscr{R}$. Assume now that we have chosen integers $g_{j}, j=0, \ldots, n-1$. In choosing $g_{n}$, we will use an auxilliary surface $\mathscr{V}=\mathscr{V}_{n}$ defined as follows: Start with the surface $\mathscr{R}_{n-1}$ constructed as described earlier using $g_{0}, g_{1}, \ldots, g_{n-1}$. In all subsequent generations, however, paste on only sheets which are copies of $\Omega_{n}$. The surface $\mathscr{V}$ is the directed limit of the resulting nested sequence of surfaces and has a natural projection $\pi_{n}$ to the unit disc. (It is not difficult to show that $\mathscr{V}$ is the image surface of an inner function, but that will not be needed here.)

Now for the choice of $g_{n}$. Choose small closed discs $D_{k}$ lying in the open unit disc, one centered on each of the points of $E_{n}$, so that $0 \notin \bigcup D_{j}$ and so that

$$
\omega\left(0, \bigcup D_{j}, D \backslash \bigcup D_{j}\right)<\frac{1}{2 n}
$$

where $\omega$ indicates harmonic measure. This is possible because $E_{n}$, being a finite set, has logarithmic capacity zero. Let $F$ be the finitely connected plane region $D \backslash \bigcup D_{j}$ and let $V$ denote the open set $\pi_{n}^{-1}(F) \subseteq \mathscr{V}$. From our construction of $\mathscr{V}$ one sees that $V$ with the projection $\left.\pi_{n}\right|_{V}$ is a regular smooth covering surface of $F$ (see $[1, \mathrm{I} .14 \mathrm{~F}]$ ). It is well known that in this situation, a Brownian path starting at $w_{0}$ in $V$ has the same probability of exiting $V$ at a point of its ideal boundary lying over $T$ as a Brownian path starting at $0=\pi_{n}\left(w_{0}\right)$ in $F$ has of exiting $F$ in $T$. But (1) implies that this latter probability is at least $1-\frac{1}{2 n}$. Furthermore, every Brownian path which exits $V$ over $T$ will have finite winding number about each of the discs $D_{j}$. Thus it will, during its wanderings before exiting $V$, visit at most some finite number of the sheets of $V$. Consequently, for a sufficiently large integer $M$, the Brownian paths on $V$ will, with probability at least $1-\frac{1}{n}$, exit over the unit circle before hitting any of the discs $D_{j}$ and before leaving the $M$ th generation of sheets of $\mathscr{V}$. Let $V^{\prime}$ be the open subset of $V$ consisting of the sheets in the first $M$ generations of the construction of $\mathscr{V}$. Brownian paths starting from $w_{0}$ will, with probability at least $1-\frac{1}{n}$, exit $V^{\prime}$ over the unit circle.

Choose $g_{n}$ so that $\sum_{j=0}^{n} g_{j} \geq M$. Then the first $M$ generations that go into the constructions of $\mathscr{R}$ and $\mathscr{V}$ are precisely the same; and in particular, $w_{0} \in V^{\prime} \subseteq \mathscr{R}_{n}$. The Brownian traveler starting at $w_{0}$ does not know whether he is in the surface $\mathscr{R}_{n}$ or $\mathscr{V}$. In either case, he will exit $V^{\prime}$ and hence the surface over the unit circle with probability at least $1-\frac{1}{n}$. Continuing inductively, we determine all the integers $g_{j}$ and complete the construction of $\mathscr{R}$.

Finally, note that the surfaces $\mathscr{R}_{j}$ are nested subsurfaces of $\mathscr{R}$. Given $\varepsilon>0$, choose $N$ so large that $\frac{1}{N}<\varepsilon$. The Brownian paths starting at $w_{0}$ exit $\mathscr{R}_{N}$ over the unit circle with probability at least $1-\varepsilon$, so they also exit $\mathscr{R}$ with at least that probability. Since $\varepsilon$ was arbitrary, almost all Brownian paths on $\mathscr{R}$ will exit over the unit circle, and we are done.

4. Properties of $f$. We know very little about the properties of $f$ in certain regards. Unlike the work on finite ranges in [11], we do not control the number of times that points in its finite range, $\bigcup E_{j}$, are taken; we know $f$ has infinitely many zeros, but it is not known where they are located nor even whether $f$ has a nontrivial singular inner factor, versus being a pure Blaschke product. Fortunately, we can still answer the last question in [12] by using a classical result of Frostman. 
For $\alpha \in D$, define a Möbius transform of $f$ by

$$
f_{\alpha}(z)=\frac{f(z)-\alpha}{1-\bar{\alpha} f(z)}, \quad z \in D .
$$

This is an inner function, and it has dense finite range

$$
\left\{w=\frac{z-\alpha}{1-\bar{\alpha} z}: z \in \bigcup E_{j}\right\} .
$$

According to the theorem of Frostman, $f_{\alpha}$ is a pure Blaschke product for all $\alpha$ outside an exceptional set $E(f)$ having logarithmic capacity zero. Thus for a typical $\alpha, f_{\alpha}$ will be a pure Blaschke product with infinitely many zeros but with values arbitrarily close to zero which are taken only finitely often.

We can determine from our construction some of the properties of the singular set of $f$, which we denote $S(f)$. This is the closed set of points of $T$ to which $f$ does not extend analytically.

First, note that almost every Brownian path on $\mathscr{R}_{f}$ will exit on a free arc of the boundary which lies over the unit circle and belongs to one of the sheets $\Omega_{j}$ used in the construction. Since $T$ is an analytic arc, one can show by the reflection principle that the mapping function $\phi$ and its projection $f=\pi \circ \phi$ will have analytic continuations to a neighborhood of that boundary point. In particular, $S(f)$ has Lebesgue measure zero. This is not the case for every inner function in $\mathscr{B}_{0}$ : Using singular measures a la Sarason, one can construct inner functions in $\mathscr{B}_{0}$ with singular set all of $T$.

On the other hand, $S(f)$ is not too small: If $E_{0}$ has at least two points, then results of Seidel and Lohwater (see Theorems 5.13 and 5.14 of [3]) imply that for every open interval $I \subseteq T$, either $S(f) \cap I$ is empty or has positive logarithmic capacity. Tom Wolff has apparently shown this to be the case for every inner function in $\mathscr{B}_{0}$, and he has suggested that 'positive capacity' can be replaced by 'Hausdorff dimension one'. Using results of Toppila [12] and the methods of J. Fernández [6], we can show that this stronger result holds for a class of functions containing $f$.

PROPOSITION. Let $g$ be an inner function whose finite range has an accumulation point in $D$. Then for every open interval $I \subseteq T$, either $S(g) \cap I$ is empty or it has Hausdorff dimension one.

Proof. Denote by $\mathscr{D}(\cdot)$ the Hausdorff dimension of a subset of $T$. Given $\varepsilon>0$, choose points $w_{1}, w_{2} \in D$ which are in the finite range of $g$ and are separated by a hyperbolic distance less than $\varepsilon$. Assume that all their preimages lie in the disc $\{|w|<R<1\}$. Let $A=\{R<|w|<1\}$ and choose a universal covering map $\psi: D \rightarrow A$ mapping $T^{+}=T \cap\{\Re z>0\}$ to $T$ and $T^{-}=T \cap\{\Re z<0\}$ to $\{|w|=R\}$. The function $G \equiv g \circ \psi$ maps $D$ into the doubly punctured disc $D \backslash\left\{w_{1}, w_{2}\right\}$. Let $\phi: D \rightarrow D \backslash\left\{w_{1}, w_{2}\right\}$ be a universal covering map. It is well known that $\phi$ is an inner function, and according to the theorem of Fernández, $\mathscr{D}(S(\phi))>\alpha(\varepsilon)$, where $\alpha(\varepsilon)$ increases to one as $\varepsilon$ goes to zero. Since $\varepsilon$ can be chosen to be arbitrarily small, it therefore suffices to prove

$$
\mathscr{D}(S(g)) \geq \mathscr{D}(S(\phi)) .
$$


Let us denote by $B$ the singular set of $g \circ \psi$ in $T^{+}$, i.e., the closed set of points at which $g \circ \psi$ fails to continue analytically. Since $\psi$ is analytic on $T^{+}$and maps it onto $T, \psi(B)=S(g)$. Therefore, (2) will follow from

$$
\mathscr{D}(B) \geq \mathscr{D}(S(\phi)) \text {. }
$$

The range of $g \circ \psi$ lies in $D \backslash\left\{w_{1}, w_{2}\right\}$, so we may factor this map through the universal covering map $\phi$; that is, there exists an analytic function $h: D \rightarrow D$ so that

$$
g \circ \psi \equiv \phi \circ h .
$$

One easily verifies by the maximum principle that since $g \circ \psi$ has modulus one almost everywhere on $T^{+}$, the same holds for $h$. Let $\Omega$ be a domain with $\Omega \cap T=$ $T^{+}$. By the Schwarz reflection principle, $h$ extends across $T^{+} \backslash B$ to a meromorphic function on $\Omega \backslash B$; moreover, $g \circ \psi \equiv \phi \circ h$ is analytic on $T^{+} \backslash B$, so $h(\Omega \backslash B)$ omits the singular set $S(\phi)$ of $\phi$. (3) follows from a theorem of Toppila (see Theorem A [6]). All these results hold locally for any interval $I$ intersecting $S(g)$, so this completes the proof.

5. Discussion. Having seen the contortions which an inner function must exhibit for membership in $\mathscr{B}_{0}$, it is tempting to think that certain features of our example are unavoidable. For instance, $f$ and the earliest example using Kahane's measure rely heavily on singular inner factors - in our case, values must be omitted on vast portions of the image surface. If all inner functions in $\mathscr{B}_{0}$ behave in an appropriate local sense like inner functions which omit values, then perhaps the suggestion of Wolff concerning the Hausdorff dimension of singular sets will follow by the methods of Fernández.

Unfortunately, inner functions and the geometry of their image surfaces remain quite mysterious. For instance, we can modify our construction of $\mathscr{R}_{f}$ in innumerable ways to obtain new examples: At each stage, as we paste on a new sheet, we can perturb the locations of the tips of the slits in that new sheet (except of course for the slit on which the pasting is being done) without invalidating our argument that we obtain an inner function in $\mathscr{B}_{0}$. We can build an inner function in $\mathscr{B}_{0}$ whose finite range has no accumulation point in $D$, or even one with empty finite range. Note however, that in all these constructions whenever a slit is used in a pasting in one generation, there are at least two branches of the associated tree along which its tip is omitted in every subsequent generation. Thus, even if the resulting function has empty finite range, it seems to have locally omitted values. Does it necessarily have a nontrivial exceptional set? Are there any inner functions in $\mathscr{B}_{0}$ whose exceptional sets are empty, i.e., indestructible Blaschke products [7]?

\section{REFERENCES}

1. Lars V. Ahlfors and Leo Sario, Riemann surfaces, Princeton Univ. Press, Princeton, N. J., 1960.

2. J. M. Anderson, On division by inner functions, Comment. Math. Helv. 54 (1979), 309-317.

3. E. F. Collingwood and A. J. Lohwater, The theory of cluster sets, Cambridge Univ. Press, Cambridge, 1966.

4. Burgess Davis, Brownian motion and analytic functions, Ann. Probab. 7 (1979), 913-932.

5. P. L. Duren, H. S. Shapiro and A. L. Shields, Singular measures and domains not of Smirnov type, Duke Math. J. 33 (1966), 247254. 
6. José L. Fernández, Singularities of inner functions, Math. Z. 193 (1986), 393-396.

7. R. McLaughlin, Exceptional sets for inner functions, J. London Math. Soc. (2) 4 (1972), 696700 .

8. George Piranian, Two monotonic, singular, uniformly almost smooth functions, Duke Math. J. 33 (1966), 255-262.

9. D. Sarason, Blaschke products in $\mathscr{B}_{0}$, Problem 6.11(old), in Linear and Complex Analysis Problem Book, 199 Research Problems, Lecture Notes in Math., vol. 1043, Springer-Verlag, Berlin and New York, 1984, pp. 337-338.

10. David A. Stegenga and Kenneth Stephenson, A geometric characterization of analytic functions with bounded mean oscillation, J. London Math. Soc. (2) 24 (1981), 243-254.

11. Kenneth Stephenson, The finite range of an inner function, Bull. London Math. Soc. 11 (1979), 300-303.

12. Sakari Toppila, On exceptional values of functions meromorphic outside a linear set, Ann. Acad. Sci. Fenn. Ser. A I Math. 5 (1980), 115-119.

13. A. Zygmund, Trigonometric series, Cambridge Univ. Press, Cambridge, 1959.

Department of Mathematics, University of Tennessee, Knoxville, TenNESSEE $37996-1300$ 